\title{
28 Research Square \\ Predictors of seizure outcome for vagus nerve stimulation in children
}

\section{Jeyul Yang}

Seoul National University Hospital

\section{Seung-Ki Kim MD}

Seoul National University Hospital

Ki Joong Kim

Seoul National University Hospital

Jong Hee Chae

Seoul National University Hospital

Byung Chan Lim

Seoul National University Hospital

Ji Hoon Phi ( $\triangle$ phi.jihoon@gmail.com )

https://orcid.org/0000-0002-9603-5843

\section{Research article}

Keywords: pediatric, epilepsy, intractable, vagus nerve stimulation

Posted Date: March 3rd, 2020

DOI: https://doi.org/10.21203/rs.3.rs-15356/v1

License: (c) (i) This work is licensed under a Creative Commons Attribution 4.0 International License.

Read Full License 


\section{Abstract}

Purpose Vagus nerve stimulation (VNS) is a palliative treatment for intractable epilepsy. While the expected outcome of VNS is well known, the clinical predictors of outcome are not clearly defined. We investigated the predictors and seizure reduction rates of VNS in pediatric patients. Methods We retrospectively reviewed medical records, radiological data, EEG, and clinical outcomes of 47 pediatric patients who underwent VNS implantation in our institution. Chi-squared tests and Fisher's exact tests were used for categorical variables, and Student's t test was applied for continuous variables. A paired T test was used for the comparison of pre- and post-insertion for each variable. Results The mean age at the time of VNS surgery was 14.7 years (range, 5-26 years). The mean interval from seizure onset to surgery was 8.3 years (range, $2-16$ years). Responders ( $\geq 50 \%$ reduction in seizure frequency) included 22 patients (47\%) at six months postoperatively and 25 patients (53\%) at one year postoperatively. The overall mean seizure frequency was reduced from 8.0 per week to 5.1 per week 12 months after implantation $(36 \%, p=0.006)$. Patients with focal onset epilepsy on electroencephalography (EEG) had 7.5 seizures per week preoperatively, which was reduced to 5.1 seizures per week postoperatively (32\%, $p=0.009)$. Patients who did not undergo resection surgery prior to VNS insertion had a reduction rate of $43 \%(p=0.006)$. Conclusions Pediatric patients with intractable epilepsy who have focal lesions in eloquent areas or unmatched ictal zones on EEG with multifocal lesions could be good candidates for VNS.

\section{Introduction}

Epilepsy is a worldwide health concern that includes children and affects developmental delay and quality of life. Despite the development of new anti-seizure drugs (ASDs) over the last few decades, $30 \%$ of patients with epilepsy remain intractable to ASD [3]. Ketogenic diet and resection surgery represent other options for intractable epilepsy patients. Recently, the role of resection surgery has been emphasized, yielding favorable outcomes [29]. However, adverse effects, such as neurological deficits or insufficient outcomes, have also been reported [27]. Thus, alternative treatments for intractable epilepsy have been constantly studied.

Vagus nerve stimulation (VNS) is a palliative treatment for intractable epilepsy and was approved by the United States of America (US) Food and Drug Administration (FDA) in 1997 [25]. To date, VNS devices have been implanted into more than 100,000 patients worldwide [12]. Numerous studies on the outcome of VNS were conducted by measuring the percentage of patients who achieved a $50 \%$ seizure frequency reduction [16]. Moreover, clinical predictors such as etiology, imaging and electroencephalography (EEG) findings for favorable outcome have been reported with univariate and multivariate analyses. However, there have been few studies regarding the seizure reduction rate after VNS implantation depending on various factors, especially in pediatric patients. In the present study, the clinical predictors and seizure reduction rates of VNS in pediatric patients with intractable epilepsy were investigated.

\section{Materials And Methods}


We retrospectively reviewed medical records, radiological data, EEG, and clinical outcomes of pediatric patients who underwent VNS implantation in our institution from January 2006 to January 2018. Medical records were reviewed for demographic data, age at onset, age at surgery, duration of epilepsy, seizure types, frequency of seizures and the number of ASDs. The present study was approved by the institutional review board at our institution. Informed consent was waivered by the IRB since this study is a retrospective study.

All patients underwent brain MRI and surface EEG prior to surgery. MRI findings were categorized into normal, focal lesions, multifocal lesions and diffuse lesions. A focal lesion was defined as a lesion that was localized in only one brain lobe. A multifocal lesion was defined as a lesion that was localized in two lobes. A diffuse lesion was defined as a lesion found in more than two lobes.

The predominant type of epilepsy was divided into three categories based on EEG monitoring, and those were focal, generalized and combined generalized and focal. EEG findings and the type of epilepsy were diagnosed by pediatric neurologists. Daily ASDs that were prescribed by pediatric neurologists were counted.

The etiology of epilepsy was categorized into structural, genetic, infectious, metabolic, immune and unknown [21]. Candidates for VNS insertion surgery were selected among patients who failed to demonstrate seizure control after adequate treatment with ASDs regardless of the presence of a lesion, type of epilepsy, or etiology. Surgical candidates were screened for whether they should receive resection surgery or VNS insertion surgery, and VNS insertion was performed if resection surgery was not indicated or if legal guardians preferred VNS insertion.

All VNS insertion surgeries were performed as a standard method and were performed on the left side with no exceptions [11]. Patients were discharged one day after surgery. The output current was initiated as $0.25 \mathrm{~mA}$, at a frequency of $30 \mathrm{~Hz}$ and a width of $500 \mathrm{~Hz} 2$ weeks after VNS insertion. Adjustment was performed in accordance with the guidelines of Cyberonics Inc. (Houston, TX) at the outpatient clinic.

Surgical outcomes were analyzed 6 and 12 months after implantation. A responder was defined as a patient who achieved more than a $50 \%$ reduction in seizure frequency [26]. Chi-squared tests and Fisher's exact tests were used for categorical variables, and Student's t test was applied for continuous variables. A paired $T$ test was used for the comparison of pre- and post-insertion for each variable. $P$ values of 0.05 or less were considered significant. IBM SPSS Statistics version 23 (Chicago, IL, USA) was used for statistical analyses.

\section{Results}

A total of 56 patients received VNS implantation in our institution during the study period. Nine patients were excluded from the outcome analyses: two patients who had to have their devices removed immediately postoperatively due to deep layer infection, six patients who were lost to follow-up within six 
months, and one patient who had additional resection surgery within six months after VNS insertion. Therefore, a total of 47 consecutive patients were included in the outcome analyses (Figure 1).

The demographic characteristics are shown in table 1. The mean age at seizure onset was 6.4 years (range, $0.2-15$ years), and the mean age at VNS surgery was 14.7 years (range, 5-26 years). The mean interval of epilepsy from onset to surgery was 8.3 years (range, 2-16 years). Twenty-five patients were male (53\%), and 22 patients were female (47\%). The mean number of preoperative ASDs was 4.2 per day (range, 1-7). The mean follow-up duration was 58.5 months (range 12-156). The mean number of ASDs was kept steady, from 4.1 per day preoperatively to 4.2 per day at one postoperative year $(p=0.371)$.

Normal MRI findings accounted for 22 patients (51\%), whereas focal, multifocal and diffuse lesions accounted for six (13\%), seven (15\%), and 12 patients (26\%), respectively. Thirty-six patients showed focal onset epilepsy on EEG. Regarding etiology, nine patients (19\%) had structural lesions, five patients $(11 \%)$ had genetic backgrounds such as SCN1A mutations or ATP1A3 mutations, nine patients had histories of central nervous system infections (19\%), one patient (2\%) had a lesion due to metabolic causes, and one patient had a lesion due to immune causes. The specific etiology was unknown in twenty-two patients.

At six months postoperatively, there were 22 patients who were responders (47\%), whereas 25 patients (53\%) were responders one year postoperatively (Figure 2 ). When the postoperative one-year responder was analyzed, the age at seizure onset was 6.9 years, the age at operation was 14.6 years, and the interval from epilepsy onset to surgery was 7.7 years. There was no statistically significant difference in the demographic characteristics between the responder and nonresponder. However, responders showed a tendency of later onset of seizure and a shorter interval from seizure onset to VNS implantation than nonresponders.

Concerning MRI findings, five out of six patients (83\%) with a focal lesion became a responder one year postoperatively, whereas three out of seven patients with multifocal (43\%) and five out of 12 patients with diffuse lesions (42\%) became responders. When analyzed with EEG findings, 4 out of 7 (57\%) generalized seizure patients and 19 out of $36(53 \%)$ focal seizure patients showed a response. Six out of nine (67\%) patients with a history of infection, and one out of four patients with a history of trauma had a response to VNS according to the etiology. Half of the patients with metabolic and genetic backgrounds were responders. Seven patients $(15 \%)$ received resection or disconnection surgery prior to VNS implantation, and those were two temporal lobectomies, two total callosotomies, two focal cortical dysplasia removals, and one tumor (pleomorphic xanthoastrocytoma) removal. Three of five $(60 \%)$ patients with a history of resection became responders. However, there was no statistically significant factor for better responses in terms of MRI, EEG, etiologies and resection history due to the small numbers of patients in each group.

The overall mean seizure frequency was reduced from 8.0 per week to 5.1 per weak $(36 \%, p=0.006$, Figure 2). The mean seizure frequency reduction rate was analyzed for the same variables used for analyses of $\geq 50 \%$ responders (Table 2 ). Of all variables, focal onset epilepsy on EEG and no prior resection surgery were statistically significant for seizure frequency reduction. Patients with focal onset epilepsy on EEG 
had 7.5 seizures per week preoperatively, which was reduced to 5.1 seizures per week $(32 \%, p=0.009)$. Likewise, the seizure frequency reduction rate was $43 \%$ in patients who had not undergone previous resection $(p=0.006)$.

Seven patients (9\%) were able to reduce their ASDs by a mean of 1.4 ASD per patient. The characteristics and comparisons of ASD reduction and nonreduction groups are shown in table 3 . The ASD reduction group demonstrated a shorter mean interval from epilepsy onset to operation than the nonreduction group. Four patients had normal MRI findings, and five patients had focal onset seizures on EEG. However, no statistical significance was obtained for the factors.

After VNS implantation, four (9\%) patients complained of hoarseness and/or cough. Symptoms were managed by output current adjustment, and all were transient. Infection occurred in three patients $(6 \%)$ when including the two patients initially excluded from the analyses because of early deep layer infection and VNS removal. The other patient could be managed by oral antibiotics without device removal.

The VNS battery was discharged in ten patients. The patients were consulted as to whether to maintain the VNS. Seven patients chose to keep their VNS treatment and underwent a battery change. One of the three patients who decided to discontinue VNS treatment remained in a seizure-free state for four years. The other two patients had minimal responses.

\section{Discussion}

The concept of stimulating the vagus nerve electronically to control seizures dates back to the late 19th century. It took nearly a century thereafter for the medical society to accept clinical application of the concept. The prototype of the currently used VNS device appeared in 1987, and the first human insertion of the device was performed in 1988 [17]. Numerous papers thereafter have reported that the outcome of VNS shares a common result with $a \geq 50 \%$ reduction rate in approximately $50 \%$ of patients [31]. The results seem to be similar in children [6]. In addition, the proportion of responders correspondingly increased as the follow-up duration increased $[5,22]$. This is in line with our result, which also showed an increase from $47 \%$ to $53 \%$ of responders at six months and one year postoperatively.

Previous surveys have attempted to document predictors of outcome regarding various variables such as age and seizure type. However, the results differ across papers. VNS has been shown to be effective in intractable focal onset epilepsy, generalized onset epilepsy, and epileptic syndromes such as LennoxGastaut syndrome (LGS) and Rett syndrome $[30,8,6]$. Studies in children with a mean age of 11.1 years old showed a reduction rate of $59 \%$ with a mean duration of VNS therapy of 5.2 years [5]. Because of the broad indications for and unknown mechanism of VNS, a predictor of better outcome has been an issue.

Age at implantation, duration from onset to implantation and seizure types have been repeatedly presented as predictors of favorable outcomes in VNS. Ghaemi et al. reported that younger age at implantation was associated with better outcomes [10]. A shorter duration of epilepsy was also reported as a factor for a favorable outcome [19]. Conflicting results coexist, however, such that older age at 
implantation and a longer duration of epilepsy are beneficial [14]. In our study, there was no statistically significant predictor of outcome in the $\geq 50 \%$ responder analysis. Several studies also could not determine the predictors of outcome based on demographic characteristics [23,32]. This is probably due to the ambiguous mechanism of VNS and widespread projection fibers of the nucleus solitarius tract, which is a transfer center of the vagus nerve [20]. Variable mechanisms have been proposed for VNS, such as cerebral blood flow changes, increases in neurotransmitters, and effects of inflammation $[2,28,18,9]$. In other words, no one particular factor seems to explain the mechanism.

In contrast to the responder analysis, analysis of the mean seizure reduction frequency yielded some statistically significant factors. Our overall seizure frequency reduction rate was in line with a metaanalysis of 1,798 patients that showed a seizure reduction rate of $36.2 \%$ (range, $3-12$ months) after surgery [6]. In addition, our results showed a statistically significant mean reduction rate of $32 \%$ for focal onset epilepsy on EEG. This corresponds to the US FDA opinion that VNS is approved for focal seizures $[13,25]$.

One interesting finding in our study is that patients with no history of resection or disconnection surgery had a significant reduction rate of $43 \%$, whereas patients with the history showed minimal effects. VNS implantation with such a history was done because of seizure recurrences. Seizure recurrences in the patients who have history of resection or disconnection surgery mean that ictal zone is ambiguous or exists somewhere else that could not be exactly identified. Amar et al. reported the outcome of VNS after failed cranial surgery for epilepsy, and patients with a history of failed cranial surgery had a lower response rate compared to patients without prior epilepsy surgery [1]. The factors of failure were physiological and anatomic limitations, such as the presence of an eloquent cortex or the improper identification of true lesions [1]. Hence, along with our results, patients with intractable epilepsy who have focal lesions in eloquent areas, who have multifocal lesions that are not removable, or who demonstrate unclear ictal zones on EEG could be good candidates for VNS.

Previous papers have reported VNS in relation to ASD reduction. One study reported that ASD reduction occurred in $43 \%$ of VNS patients, with an average of 0.43 ASDs per patient [24]. ASD reduction is especially important in pediatrics, considering the adverse effects of long-term use. Prolonged use of ASDs is well known to result in cognitive and psychological dysfunction $[7,15]$. The characteristics of the reduction group in our study correspond to other analyses in terms of an older epilepsy onset, shorter interval and focal onset epilepsy in EEG.

It is intriguing that $70 \%$ of patients chose to maintain their therapy by changing batteries. The reasons for the decision were improved alertness, decreased seizure intensity, and overall improvements in quality of life. One patient had improvements in subjective cognitive function rather than seizure control and chose to maintain the treatment. This implies that indications of VNS are not limited to epilepsy but can be applied to other conditions. To date, VNS has been suggested to show improvements in alertness and depression $[22,4]$. 
There are some limitations to our study. First, this was a retrospective study with a relatively small number of patients enrolled. Second, seizure outcome could be affected by various other factors, especially changes in ASD doses. However, the study focused purely on children with intractable epilepsy. To overcome the low statistical power, we added an analysis of mean seizure frequency reduction to compensate for the $\geq 50 \%$ response criteria.

\section{Conclusion}

VNS is a palliative treatment, and various predictors of outcome are still under debate. Considering that VNS is not a curative treatment, selecting patients who will benefit from the treatment is important.

Based on a low morbidity rate and relatively easy procedure for neurosurgeons to perform, VNS could be a promising option for pediatric intractable epilepsy, especially for focal epilepsy patients who carry risks of undergoing resection or disconnection epilepsy surgery for various reasons, such as an ictal zone in eloquent areas or uncertain ictal zones. Moreover, patients may also benefit from reducing ASDs in addition to seizure reduction regarding the consensus that the efficacy of VNS increases over time. For pediatric candidates, it is advisable to perform VNS insertion sooner to maximize the efficacy of VNS and to prevent side effects of long-term ASD use.

\section{Declarations}

\section{Declarations of interest: none}

Acknowledgment: This Research was supported by National Evidence-based Healthcare Collaborating Agency (NECA) funded by the Ministry of Health and Welfare (grant number: HC19C0180)

Data Availability: The datasets generated during and/or analysed during the current study are available from the corresponding author on reasonable request

\section{References}

1. Amar AP, Apuzzo ML, Liu CY (2004) Vagus nerve stimulation therapy after failed cranial surgery for intractable epilepsy: results from the vagus nerve stimulation therapy patient outcome registry. Neurosurgery 55:1086-1093

2. Ben-Menachem E, Hamberger A, Hedner T, Hammond E, Uthman B, Slater J, Treig T, Stefan H, Ramsay R, Wernicke J (1995) Effects of vagus nerve stimulation on amino acids and other metabolites in the CSF of patients with partial seizures. Epilepsy Res 20:221-227

3. Chen Z, Brodie MJ, Liew D, Kwan P (2018) Treatment outcomes in patients with newly diagnosed epilepsy treated with established and new antiepileptic drugs: A 30-year longitudinal cohort study. JAMA Neurology 75:279-286. doi:10.1001/jamaneurol.2017.3949 
4. Elger G, Hoppe C, Falkai P, Rush AJ, Elger CE (2000) Vagus nerve stimulation is associated with mood improvements in epilepsy patients. Epilepsy Res 42:203-210.

doi:https://doi.org/10.1016/S0920-1211(00)00181-9

5. Elliott RE, Rodgers SD, Bassani L, Morsi A, Geller EB, Carlson C, Devinsky O, Doyle WK (2011) Vagus nerve stimulation for children with treatment-resistant epilepsy: a consecutive series of 141 cases. J Neurosurg Pediatr 7:491-500

6. Englot DJ, Chang EF, Auguste KI (2011) Vagus nerve stimulation for epilepsy: a meta-analysis of efficacy and predictors of response: a review. J Neurosurg 115:1248-1255

7. Farwell JR, Lee YJ, Hirtz DG, Sulzbacher SI, Ellenberg JH, Nelson KB (1990) Phenobarbital for febrile seizures-effects on intelligence and on seizure recurrence. N Engl J Med 322:364-369

8. Frost M, Gates J, Helmers SL, Wheless JW, Levisohn P, Tardo C, Conry JA (2001) Vagus Nerve Stimulation in Children with Refractory Seizures Associated with Lennox-Gastaut Syndrome. Epilepsia 42:1148-1152. doi:doi:10.1046/j.1528-1157.2001.23900.x

9. Garnett E, Nahmias C, Scheffel A, Firnau G, Upton AJP, Electrophysiology C (1992) Regional cerebral blood flow in man manipulated by direct vagal stimulation. 15:1579-1580

10. Ghaemi K, Elsharkawy AE, Schulz R, Hoppe M, Polster T, Pannek H, Ebner A (2010) Vagus nerve stimulation: outcome and predictors of seizure freedom in long-term follow-up. Seizure 19:264-268. doi:10.1016/j.seizure.2010.03.002

11. Giordano F, Zicca A, Barba C, Guerrini R, Genitori L (2017) Vagus nerve stimulation: Surgical technique of implantation and revision and related morbidity. Epilepsia 58:85-90

12. Johnson RL, Wilson CG (2018) A review of vagus nerve stimulation as a therapeutic intervention. J Inflamm Res 11:203-213. doi:10.2147/JIR.S163248

13. Kim M-J, Yum M-S, Kim E-H, Lee Y-J, Lee J, Hong S, You SJ, soon Hwang Y, Ko T-S (2017) An interictal EEG can predict the outcome of vagus nerve stimulation therapy for children with intractable epilepsy. Childs Nerv Syst 33:145-151

14. Labar D (2004) Vagus nerve stimulation for 1 year in 269 patients on unchanged antiepileptic drugs. Seizure 13:392-398

15. Lagae L (2006) Cognitive side effects of anti-epileptic drugs: the relevance in childhood epilepsy. Seizure 15:235-241

16. Panebianco M, Zavanone C, Dupont S, Restivo DA, Pavone A (2016) Vagus nerve stimulation therapy in partial epilepsy: a review. Acta Neurol Belg 116:241-248. doi:10.1007/s13760-016-0616-3

17. Penry JK, Dean JC (1990) Prevention of intractable partial seizures by intermittent vagal stimulation in humans: preliminary results. Epilepsia 31:S40-S43

18. Raedt R, Clinckers R, Mollet L, Vonck K, El Tahry R, Wyckhuys T, De Herdt V, Carrette E, Wadman W, Michotte $Y$ (2011) Increased hippocampal noradrenaline is a biomarker for efficacy of vagus nerve stimulation in a limbic seizure model. J Neurochem 117:461-469 
19. Renfroe JB, Wheless JW (2002) Earlier use of adjunctive vagus nerve stimulation therapy for refractory epilepsy. Neurology 59:S26-S30

20. Rutecki P (1990) Anatomical, physiological, and theoretical basis for the antiepileptic effect of vagus nerve stimulation. Epilepsia 31:S1-S6

21. Scheffer IE, Berkovic S, Capovilla G, Connolly MB, French J, Guilhoto L, Hirsch E, Jain S, Mathern GW, Moshé SL (2017) ILAE classification of the epilepsies: position paper of the ILAE Commission for Classification and Terminology. Epilepsia 58:512-521

22. Serdaroglu A, Arhan E, Kurt G, Erdem A, Hirfanoglu T, Aydin K, Bilir E (2016) Long term effect of vagus nerve stimulation in pediatric intractable epilepsy: an extended follow-up. Childs Nerv Syst 32:641646

23. Sherman EM, Connolly MB, Slick DJ, Eyrl KL, Steinbok P, Farrell K (2008) Quality of life and seizure outcome after vagus nerve stimulation in children with intractable epilepsy. J Child Neurol 23:991998

24. Tatum WO, Johnson KD, Goff S, Ferreira JA, Benbadis SR, Vale FL (2001) Vagus nerve stimulation and drug reduction. Neurology 56:561-563. doi:10.1212/wnl.56.4.561

25. Terry Jr RS (2014) Vagus nerve stimulation therapy for epilepsy. In: Epilepsy Topics. InTech,

26. Uthman BM, Wilder BJ, Penry JK, Dean C, Ramsay RE, Reid SA, Hammond EJ, Tarver WB, Wernicke JF (1993) Treatment of epilepsy by stimulation of the vagus nerve. Neurology 43:1338-1338. doi:10.1212/wnl.43.7.1338

27. Van Schooneveld MMJ, Braun KPJ (2013) Cognitive outcome after epilepsy surgery in children. Brain Dev 35:721-729. doi:https://doi.org/10.1016/j.braindev.2013.01.011

28. Walker BR, Easton A, Gale K (1999) Regulation of limbic motor seizures by GABA and glutamate transmission in nucleus tractus solitarius. Epilepsia 40:1051-1057

29. Wiebe S, Blume WT, Girvin JP, Eliasziw M (2001) A randomized, controlled trial of surgery for temporal-lobe epilepsy. N Engl J Med 345:311-318

30. Wilfong AA, Schultz RJ (2006) Vagus nerve stimulation for treatment of epilepsy in Rett syndrome. Dev Med Child Neurol 48:683-686

31. Yang J, Phi JH (2019) The Present and Future of Vagus Nerve Stimulation. Journal of Korean Neurosurgical Society 62:344

32. Yu C, Ramgopal S, Libenson M, Abdelmoumen I, Powell C, Remy K, Madsen JR, Rotenberg A, Loddenkemper T (2014) Outcomes of vagal nerve stimulation in a pediatric population: a single center experience. Seizure 23:105-111

\section{Tables}

Table 1. Demographic characteristics 


\begin{tabular}{|c|c|c|c|c|}
\hline \multirow{2}{*}{ ye, years $\pm S D$} & \multicolumn{4}{|c|}{ Total $(\mathrm{N}=47)_{\text {Responder }}{ }^{\mathrm{a}}(\mathrm{N}=25)$ Nonresponder $(\mathrm{N}=22) \mathrm{P}$-value } \\
\hline & & & & \\
\hline әрsy onset & $6.4 \pm 4.2$ & $6.9 \pm 4.2$ & $5.8 \pm 4.1$ & .330 \\
\hline \multirow{5}{*}{$\begin{array}{l}\text { antation } \\
\text { l from epilepsy onset to implantation }\end{array}$} & $14.7 \pm 5.7$ & $14.6 \pm 5.1$ & $14.7 \pm 6.4$ & .959 \\
\hline & $8.3 \pm 5.4$ & $7.7 \pm 4.3$ & $9.1 \pm 6.4$ & .391 \\
\hline & & & & .861 \\
\hline & 25 & 13 & 12 & \\
\hline & 22 & 12 & 10 & \\
\hline \multirow{3}{*}{$\begin{array}{l}\text { lmber of preop. seizure medication } \pm \text { SD } \\
\text { ling }(\%)\end{array}$} & $4.2 \pm 1.3$ & $4.2 \pm 1.5$ & $4.1 \pm 1.0$ & .949 \\
\hline & & & & .387 \\
\hline & 22 & 12 & 10 & \\
\hline \multirow{2}{*}{ cal lesion } & 6 & 5 & 1 & \\
\hline & 7 & 3 & 4 & \\
\hline lesion & 12 & 5 & 7 & \\
\hline \multirow{2}{*}{$\begin{array}{l}\text { epilepsy by EEG finding } \\
\text { nset }\end{array}$} & & & & .157 \\
\hline & 36 & 19 & 17 & \\
\hline \multirow{3}{*}{$\begin{array}{l}\text { lized onset } \\
\text { : generalized onset }\end{array}$} & 7 & 4 & 3 & \\
\hline & 2 & 0 & 2 & \\
\hline & 2 & 2 & 0 & \\
\hline \multirow{2}{*}{$\begin{array}{l}\text { tiology } \\
\text { ral }\end{array}$} & & & & .649 \\
\hline & 7 & 2 & 5 & \\
\hline 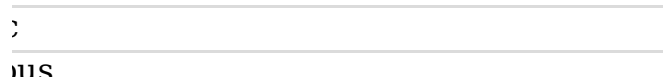 & 5 & 3 & 2 & \\
\hline \multirow{2}{*}{$\begin{array}{l}\text { ius } \\
\text { lic }\end{array}$} & 9 & 6 & 3 & \\
\hline & 1 & 0 & 1 & \\
\hline 3 & 1 & 1 & 0 & \\
\hline \multirow[t]{2}{*}{ s operation } & & & & .291 \\
\hline & 40 & 22 & 18 & \\
\hline on surgery & 5 & 3 & 2 & \\
\hline tomy & 2 & 0 & 2 & \\
\hline
\end{tabular}

s who achieved $\geq 50 \%$ reduction of seizure frequency at 1 year post- VNS insertion

Table 2. Mean seizure frequency reduction per week 


\begin{tabular}{|c|c|c|c|c|}
\hline$\overline{\text { riable }}$ & Pre-implantation & 1 year post-implantation & Mean reduction rate (\%) & P-value \\
\hline an seizure frequency \pm SD & $8.0 \pm 8.9$ & $5.1 \pm 6.6$ & 36 & .006 \\
\hline RI finding & & & & .149 \\
\hline ormal & $6.6 \pm 8.3$ & $5.3 \pm 6.9$ & 20 & .274 \\
\hline ical lesion & $7.9 \pm 8.0$ & $4.4 \pm 4.4$ & 44 & .305 \\
\hline ultifocal lesion & $11.6 \pm 7.5$ & $7.0 \pm 7.7$ & 40 & .085 \\
\hline ffuse lesion & $8.6 \pm 11.5$ & $4.0 \pm 6.8$ & 53 & .119 \\
\hline $\begin{array}{l}\text { pe of epilepsy by EEG finding } \\
\text { ical onset }\end{array}$ & $7.5 \pm 7.9$ & $5.1 \pm 7.2$ & 32 & .009 \\
\hline neralized onset & $14.5 \pm 12.8$ & $7.8 \pm 9.3$ & 46 & .086 \\
\hline cal \& generalized onset & $2.5 \pm 0.7$ & $8.8 \pm 2.5$ & -352 & .220 \\
\hline Jrmal & $0.3 \pm 0.0$ & $0.1 \pm 0.1$ & 67 & .156 \\
\hline own etiology & & & & \\
\hline ructural & $9.9 \pm 10.7$ & $7.6 \pm 11.7$ & 23 & .158 \\
\hline netic & $7.8 \pm 9.3$ & $4.6 \pm 5.9$ & 41 & .306 \\
\hline fectious & $2.9 \pm 4.3$ & $2.8 \pm 4.7$ & 3 & .723 \\
\hline etabolic & 3 & 1 & & - \\
\hline imune & 21 & 21 & & - \\
\hline נvious operation & & & & \\
\hline one & $8.0 \pm 8.7$ & $4.6 \pm 5.4$ & 43 & .006 \\
\hline sionectomy & $11.5 \pm 12.4$ & $11.3 \pm 12.6$ & 2 & .284 \\
\hline Illosotomy & $0.6 \pm 0.2$ & $1.0 \pm 0.0$ & -40 & .205 \\
\hline
\end{tabular}

Table 3. Comparison between anti-seizure drug reduction group and nonreduction group 


\begin{tabular}{|c|c|c|c|}
\hline \multirow{2}{*}{ ye, years $\pm S D$} & \multicolumn{3}{|c|}{ Reduction ( $\mathrm{N}=7$ )Non-reduction $(\mathrm{N}=40)$ P-value } \\
\hline & & & \\
\hline \multirow[t]{2}{*}{ әpsy onset } & $8.3 \pm 5.0$ & $6.1 \pm 4.0$ & .194 \\
\hline & $14.4 \pm 5.0$ & $14.8 \pm 5.9$ & .901 \\
\hline \multirow[t]{4}{*}{ l from epilepsy onset to operation } & $6.1 \pm 4.4$ & $8.7 \pm 5.5$ & .247 \\
\hline & & & .690 \\
\hline & 3 & 18 & \\
\hline & 4 & 22 & \\
\hline \multirow[t]{2}{*}{$\overline{\text { ling }}$} & & & .454 \\
\hline & 4 & 18 & \\
\hline sion & 1 & 5 & \\
\hline cal lesion & 1 & 6 & \\
\hline lesion & 1 & 11 & \\
\hline \multicolumn{2}{|l|}{ epilepsy by EEG finding } & & .639 \\
\hline nset & 5 & 31 & \\
\hline lized onset & 2 & 5 & \\
\hline \multirow{2}{*}{ generalized onset } & 0 & 2 & \\
\hline & 0 & 2 & \\
\hline 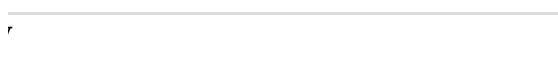 & & & .750 \\
\hline ral & 1 & 6 & \\
\hline 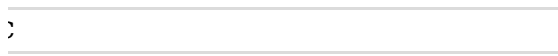 & 1 & 4 & \\
\hline ius & 3 & 6 & \\
\hline lic & 0 & 1 & \\
\hline כ & 0 & 1 & \\
\hline $\mathrm{vn}$ & 2 & 22 & \\
\hline
\end{tabular}

Figures 
Patients who underwent VNS insertion from January 2006 to January 2018 $(\mathrm{N}=56)$
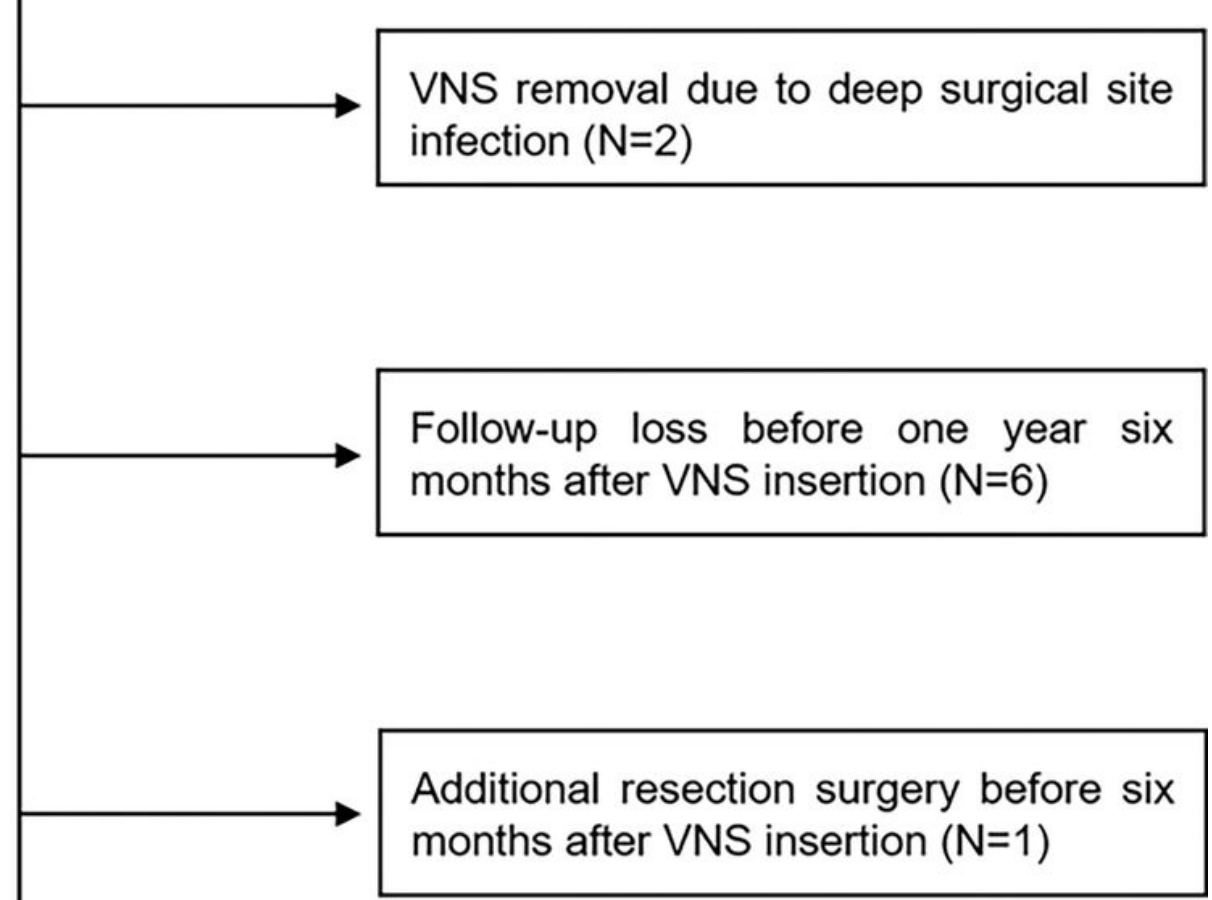

Total VNS insertion cases at post-operative 1 year $(\mathrm{N}=47)$

\section{Figure 1}

Flow chart of patients included in the study. Among 56 patients who underwent VNS insertion, a total of 47 patients were enrolled in the study. 


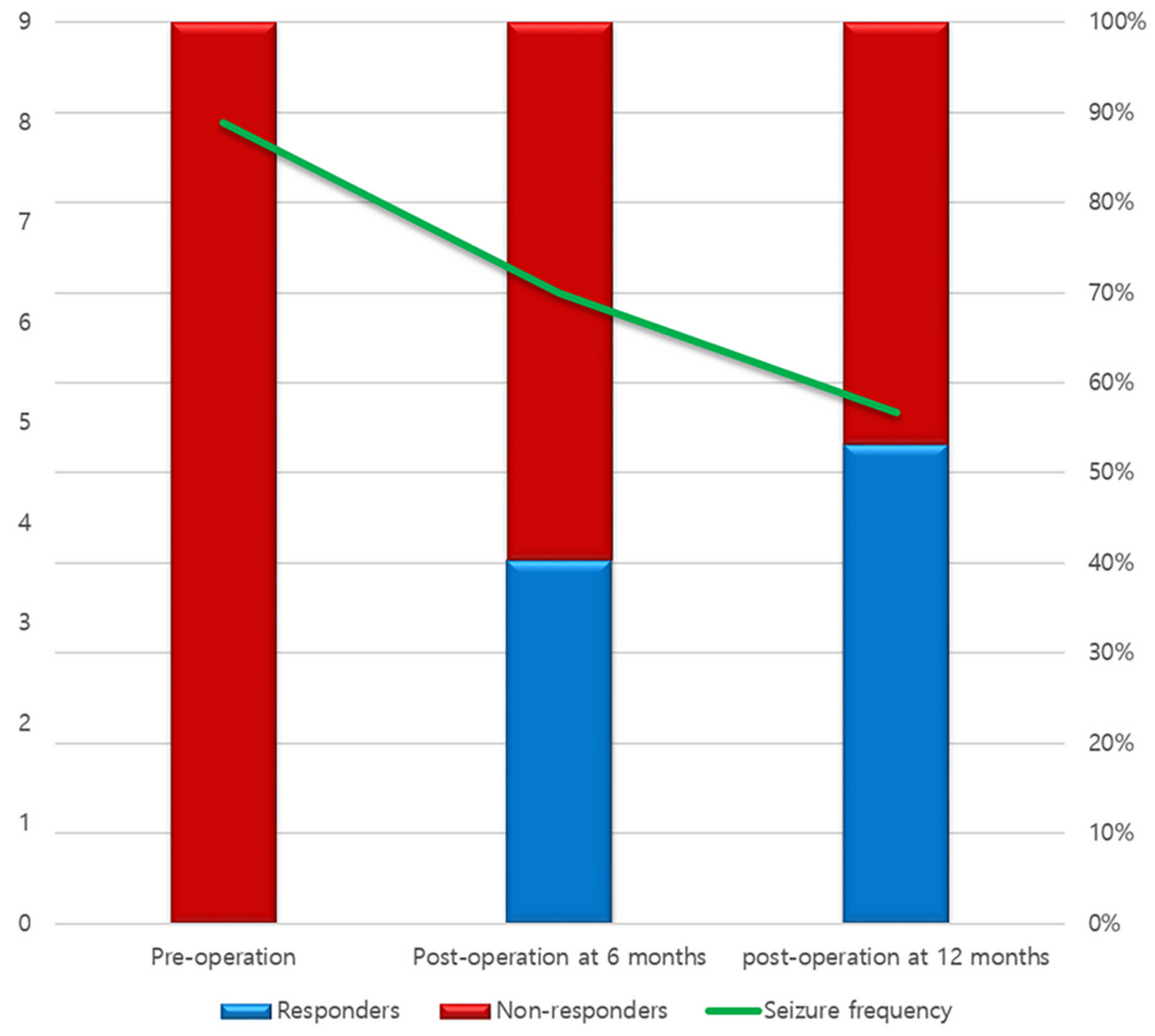

Figure 2

Graph showing reductions and seizure frequency changes during the follow-up period. The left $Y$-axis is the mean seizure frequency, and the right $Y$-axis is the percentage of the responder. 